\title{
基于非特异性蛋白酶连续酶解的蛋白质全序列测定方法
}

\author{
杨超 $a, b$ 单亦初 $*, a$ 张玮杰 $a, b$ 戴忠鹏 $a$ \\ 张丽华*, $a$ 张玉奎 $a$ \\ ( ${ }^{a}$ 中国科学院大连化学物理研究所 中国科学院分离分析化学重点实验室 大连 116023) \\ ( $b$ 中国科学院大学 北京 100049)
}

\begin{abstract}
摘要 对蛋白质全序列进行测定, 有助于分析蛋白质的结构, 揭示蛋白质的生物学功能. 针对目前基于质谱的蛋白质 测序流程中使用特异性蛋白酶酶解产生的肽段种类少、重叠度低、序列拼接困难等问题，发展了一种基于非特异性蛋 白酶连续酶解的蛋白质全序列测定方法. 构建了连续酶解装置, 并使用多种非特异性蛋白酶对蛋白质进行连续酶解. 利用非特异性蛋白酶酶解位点的非特异性、不同的酶解时间以及不同种类蛋白酶酶解产生肽段的互补性，提高蛋白质 酶解肽段的种类和重叠度, 并发展了蛋白质序列拼接算法对液相色谱质谱联用(LC-MS/MS)和从头测序获得的肽段序 列进行拼接. 将此方法应用于牛血清白蛋白和单克隆抗体赫赛汀的全序列测定, 在不考虑亮氨酸和异亮氨酸的情况下, 对牛血清白蛋白和赫赛汀轻链的测序准确度达到 $100 \%$ ，赫赛汀重链的测序准确度为 $99.7 \%$.

关键词 非特异性蛋白酶; 连续酶解; 序列拼接; 全序列测定
\end{abstract}

\section{Full-length Protein Sequencing Based on Continuous Digestion Using Non-specific Proteases}

\author{
Chao Yang ${ }^{a, b} \quad$ Yi-Chu Shan*,a Wei-Jie Zhang ${ }^{a, b} \quad$ Zhong-Peng Dai $^{a}$ \\ Li-Hua Zhang*,a Yu-Kui Zhang ${ }^{a}$ \\ ( ${ }^{a}$ CAS Key Laboratory of Separation Science for Analytical Chemistry, Dalian Institute of Chemical Physics, \\ Dalian 116023, China) \\ ( ${ }^{b}$ University of Chinese Academy of Sciences, Beijing 100049, China)
}

\begin{abstract}
Determining the complete sequence of the protein is helpful to analyze the structure of the protein and reveal the biological function of the protein. In traditional "bottom-up" proteomic strategy, database searching is used to identify sequences of peptides and proteins analyzed by liquid chromatography-tandem mass spectrometry (LC-MS/MS). It is impossible to identify proteins with unknown sequences through database searching, so de novo sequencing is essential for protein characterization. To increase the accuracy and coverage of protein sequencing, a de novo protein sequencing method based on continuous digestion using various non-specific proteases has been developed. A continuous digestion device was constructed, and a variety of non-specific proteases were used to continuously digest the protein. Taking advantage of the non-specific cleavage sites of non-specific proteases, the complementarity of peptides produced at different time and by different kinds of proteases, the type and overlapping degree of digested peptides were improved. The sequence coverage of peptides after continuous digestion by each protease can reach $100 \%$. Finally, a sequence assembly algorithm was developed to assemble the peptides obtained by de novo sequencing. At first, the candidate peptide sequences were splitted into sequence tags which contain 7 amino acids, and then the most frequently occurring sequence tag was chosen as the seed sequence. Afterwards, the seed sequence was automatically or manually extended to the $\mathrm{N}$-terminal end and C-terminal end respectively according to the scores of sequence tags. Finally, the complete protein sequence was successfully assembled. The developed method was applied to the de novo sequencing of bovine serum albumin (BSA) and monoclonal antibody Herceptin. Excluding leucine and isoleucine, full-length de novo sequencing was achieved with $100 \%$ accuracy for BSA and Herceptin light chain. Accuracy of the sequenced Herceptin heavy chain was $99.7 \%$. The de novo sequencing strategy based on continuous digestion of proteins using non-specific proteases can be applied to de novo sequencing of proteins with unknown sequences or quality control of monoclonal antibody drugs.

Keywords non-specific protease; continuous digestion; sequence assembly; full-length sequencing
\end{abstract}

\footnotetext{
*E-mail: shanyichu@dicp.ac.cn; lihuazhang@dicp.ac.cn

Received January 27, 2021; published March 26, 2021.

Supporting information for this article is available free of charge via the Internet at http://sioc-journal.cn.

Project supported by the Ministry of Science and Technology of China (Nos. 2017YFF0205404, 2017YFA0505004) and the National Natural Science Foundation of China (Nos. 21675153, 21725506).

项目受国家重点研发计划课题(Nos. 2017YFF0205404, 2017YFA0505004)和国家自然科学基金(Nos. 21675153, 21725506)资助.
} 


\section{1 引言}

蛋白质序列的表征是蛋白质定性分析的一个重要 部分. 蛋白质测序对蛋白质的生物功能研究 ${ }^{[1]}$ 、疾病相 关蛋白质突变体的发现 ${ }^{[2-3]}$ 和抗体药物的表征 ${ }^{[4]}$ 起着关 键作用. 随着高精度质谱的快速发展, 基于液相色谱质谱技术对蛋白质的分析鉴定已经成为蛋白质序列测 定的核心技术. 通过质谱对蛋白质分析有两种策略, 一 种是自上而下法(top-down), 指的是蛋白质不经过酶解 消化直接进入质谱中分析, 产生一级谱图和二级谱图, 再通过计算机直接鉴定蛋白质 ${ }^{[5-7]}$. 另一种是自下而上 法(bottom-up), 也称鸟枪法, 通过对蛋白质水解产物进 行 LC-MS/MS 分析, 根据肽段来对蛋白质进行鉴定 ${ }^{[8-11]}$. 自下而上的蛋白质鉴定包括多个环节, 包括蛋白质提取 分离、蛋白质样品的消化、质谱数据的采集以及质谱数 据分析, 每一个步骤都会影响到蛋白质鉴定的总体效 果. 对蛋白质测序的主要流程通常可分为两部分: 生化 实验和质谱数据分析.

蛋白质的消化是蛋白质鉴定生化实验的关键部分, 对蛋白质消化可以通过酸解或者蛋白酶酶解使蛋白质 中的酰胺键断裂, 产生肽段, 进行质谱的鉴定. 胰蛋白 酶是最常用的蛋白酶之一. 但由于蛋白质经过胰蛋白酶 酶解后产生的肽段过短、过长或者疏水性强, 在质谱中 碎裂效率低. 因此只使用胰蛋白酶消化蛋白质, 产生的 肽段序列覆盖率达不到 $100 \%$, 并且仅使用胰蛋白酶酶 解后的肽段之间没有重叠区域 ${ }^{[12]}$, 所以无法进行序列 拼接. 非特异性蛋白酶可以识别多个氨基酸残基位点, 所产生的肽段种类较特异性蛋白酶更丰富, 并且在酶解 过程中不会引入额外的修饰, 可提高蛋白质的序列覆盖 率. 对于复杂的蛋白质样品, 例如蛋白质复合物, 通过 结合特异性和非特异性蛋白酶酶解可以提高蛋白质和 翻译后修饰的鉴定覆盖率 [13-14].

基于质谱对蛋白质鉴定的算法可分为两大类: 数据 库检索和从头测序. 数据库检索是将质谱数据与数据库 进行对比和匹配获得肽段信息 ${ }^{[8]}$; 肽段从头测序不依赖 蛋白质的数据库, 直接通过谱图中的离子信息来推导肽 段的序列 ${ }^{[8,15-17]}$. 从头测序技术可以对未知序列蛋白 质、发生突变的蛋白质以及发生翻译后修饰的蛋白质进 行测序. 目前从头测序的主要算法有 PEAKS 系列[18-19]、 pNovo 系列[20-23]、PepNovo ${ }^{[24-25]} 、$ Novor $^{[26]}$ 等. 成茜等 ${ }^{[27]}$ 对 Novor, pNovo3 和 PepNovo+这三种从头测序软件对 其序列覆盖度、测序准确率、打分系统的特异性及测序 速度进行了评估. Muth 等 ${ }^{[28]}$ 评估了 Novor、PEAKS 和 PepNovo 三种测序软件在实验和模拟数据集上测序的 准确度和运行时间, 这三款软件虽然对模拟数据的测序 准确度可达到 $84 \%$, 但对高分辨率的实验数据测序准确 度最高只有 $35 \%$. 虽然目前发展的肽段从头测序算法较 多且种类不断更新, 但由于质谱谱图中存在着噪声和干 扰离子, 无法准确地识别谱图中肽段的特征碎片离
子 [29], 精准解析谱图的难度增加且测序准确率较低. 在 肽段的从头测序准确率低的情况下，使用序列拼接软件 对序列进行正确的拼接是有一定难度的. Savidor 等 ${ }^{[30]}$ 发展了一种蛋白质序列拼接算法—— “肽段标签组装” (peptide tag assembler, PTA), 通过该算法对蛋白质酸解 样品进行序列拼接. 对三种序列已知的蛋白质和一种序 列未知的单克隆抗体进行测序并拼接, 测序准确率可达 到 $99 \% \sim 100 \%$, 但抗体重链的序列有 23 个氨基酸未被 覆盖到. 同时由于在酸解过程中, 氨基酸残基会发生脱 氨基化, 天冬酰胺和谷氨酰胺分别变成天冬氨酸和谷氨 酸. 无法对序列中的天冬氨酸和谷氨酰胺进行准确的鉴 定. Tran 等 ${ }^{[11]}$ 发展了一种序列拼接方法一一蛋白质全序 列拼接(ALPS), 对两种单克隆抗体重链的测序准确度 分别为 $99.09 \%$ 和 $96.64 \%$.

本文提出了一种基于非特异性蛋白酶酶解的蛋白 质全序列测定方法. 首先通过连续酶解装置, 对蛋白质 经非特异性蛋白酶解后产生的肽段进行连续的收集, 再 通过 LC-MS/MS 对肽段进行分析, 使用从头测序软件对 肽段从头测序获得肽段序列以及对肽段序列进行拼接, 最终可得到蛋白质的完整序列.

\section{2 结果与讨论}

\section{1 原理策略}

在蛋白质从头测序的过程中, 蛋白质经过特异性 蛋白酶酶解后得到的肽段, 因为有些肽段长度过短或肽 段间序列重叠部分少，无法将这些肽段序列拼接成完整 的蛋白质的序列. 为了解决这一问题, 发展了一种使用 非特异性蛋白酶连续酶解的蛋白质测序方法.

我们首先验证了非特异性蛋白酶在不同酶解时间 产生不同种类的肽段. 为了连续收集不同时间的酶解肽 段, 设计了一个连续酶解的装置, 通过此装置对蛋白质 进行连续酶解, 可以产生种类更多的肽段, 从而增加蛋 白质序列覆盖度以及肽段之间的重叠度. 首先将目标蛋 白质与非特异性蛋白酶置于微升进样针中, 调节加热管 温度至最适酶解温度, 使用电动泵将酶与蛋白质的混合

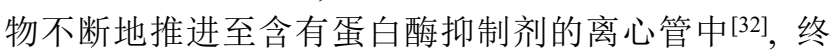
止酶解, 可以获得经过连续酶解的肽段. 这些混合肽段 经 LC-MS/MS 的分离并采集得到 raw 文件，再使用 PEAKS 软件对肽段进行从头测序, 使用我们发展的拼 接算法, 对这些从头测序得到的肽段进行分解、叠加和 延伸，最终可拼接成完整的蛋白质序列，完成蛋白质的 序列测定.

\section{2 不同酶解时间与肽段种类}

非特异性蛋白酶对蛋白质序列中酰胺键的断裂特 异性低, 酶切位点不固定具有随机性, 为验证同一种非 特异性蛋白酶在其最适酶解条件下，经过不同酶解时间 可将蛋白质酶解成不同种类和数量的肽段, 进行了以下 
实验, 并对酶解后肽段进行分析.

分别使用菠萝蛋白酶、糜蛋白酶、弹性蛋白酶、蛋 白酶 $\mathrm{K}$ 、链霉蛋白酶和嗜热菌蛋白酶对牛血清白蛋白 (BSA) 进行一次酶解. 每种蛋白酶的一次酶解的时间分 别为 $5 、 10 、 15 、 20 、 40 、 60 、 90 、 120 、 150 、 180 、 210$ 、 $240 、 270 、 300 、 330 、 360 、 390$ 和 $420 \mathrm{~min}$, 共计 18 个 时间点. 然后通过基质辅助激光解析/电离飞行时间质 谱(MALDI-TOF MS)对经不同蛋白酶酶解和不同酶解 时间产生的肽段进行鉴定. 根据谱图中的质荷比和峰强 度, 从每种蛋白酶酶解后的 18 个谱图中挑选出 5 个质荷 比和峰强度相差较大的谱图, 并统计这些谱图的酶解时 间(表 1).

表 1 BSA 由不同蛋白酶酶解的时间(min)

Table 1 The digestion time (min) for BSA to be digested by different proteases

\begin{tabular}{cccccc}
\hline 蛋白酶 & 时间 1 & 时间 2 & 时间 3 & 时间 4 & 时间 5 \\
\hline 菠萝蛋白酶 & 20 & 90 & 180 & 270 & 360 \\
糜蛋白酶 & 20 & 90 & 150 & 300 & 420 \\
弹性蛋白酶 & 20 & 120 & 180 & 240 & 420 \\
蛋白酶 K & 20 & 90 & 180 & 270 & 360 \\
链需蛋白酶 & 20 & 40 & 120 & 150 & 180 \\
嗜热菌蛋白酶 & 5 & 90 & 210 & 300 & 360 \\
\hline
\end{tabular}

对使用不同非特异性蛋白酶在不同时间的一次酶 解得到的肽段种类进行分析, 从图 1 可以看出六种非特 异性蛋白酶在不同时间产生的肽段数目都是不同的, 使 用菠萝蛋白酶、糜蛋白酶、弹性蛋白酶、蛋白酶 K、链 霉蛋白酶和嗜热菌蛋白酶分别在酶解 20、90、120、20、 20 和 $5 \mathrm{~min}$ 时产生肽段种类最多, 其中蛋白酶 $\mathrm{K}$ 可将由 583 个氨基酸组成的 BSA 酶解成 976 种不同序列的肽 段. 由糜蛋白酶和弹性蛋白酶酶解产生的肽段种类随着 酶解时间的增加先增大, 然后逐渐减少. 由菠萝蛋白 酶、蛋白酶 K、链霉蛋白酶和嗜热菌蛋白酶酶解产生的 肽段种类由多变少是由于检测时间过晚导致的.
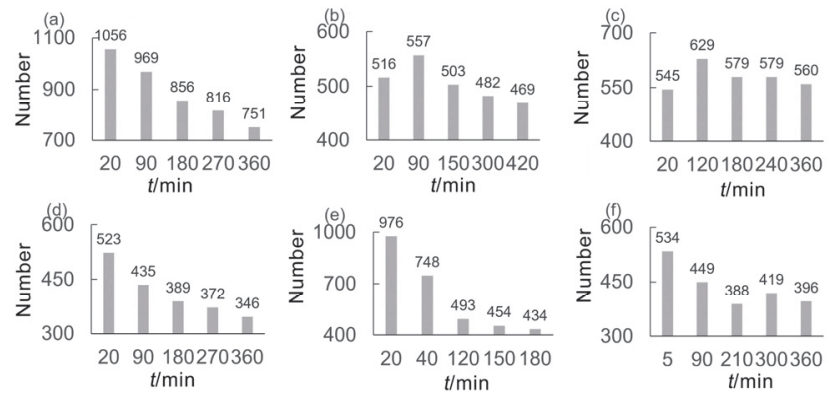

图 1 BSA 经过不同蛋白酶在不同酶解时间后所产生的肽段种类. (a) 菠萝蛋白酶. (b)糜蛋白酶. (c)弹性蛋白酶. (d) 蛋白酶 K. (e)链霉蛋白酶. (f)嗜热菌蛋白酶

Figure 1 Number of BSA peptides produced using different proteases for different digestion times. (a) bromelain. (b) chymotrypsin. (c) elastase. (d) proteinase K. (e) pronase. (f) thermolysin

对不同酶解时间产生肽段种类的交集进行分析(图
2), BSA 在蛋白酶 $\mathrm{K}$ 酶的作用下，经 20、40、120、150 和 $180 \mathrm{~min}$ 酶解后, 分别产生了序列不同的肽段. 对这 些肽段序列交集进行分析，在这五个不同酶解时间都被 鉴定到的肽段序列有 265 种; 被鉴定到四次的肽段序列 有 81 种; 被鉴定到三次的肽段序列有 122 种; 被鉴定到 两次的肽段序列有 357 种; 只被鉴定到一次的肽段有 376 种. 同样的, 对其他五种非特异性蛋白酶对 BSA 酶 解产生的肽段序列交集进行分析，可得到相似的结果， 在经过不同酶解时间酶解后，仅有一部分肽段的都能被 鉴定到. 可见，经过长时间酶解产生的肽段种类，并不 是短时间产生肽段种类的子集，蛋白质在非特异性蛋白 酶作用下会不断产生新的肽段. 因此使用非特异性蛋白 酶对蛋白质酶解, 经过不同的酶解时间所产生的肽段种 类不同.

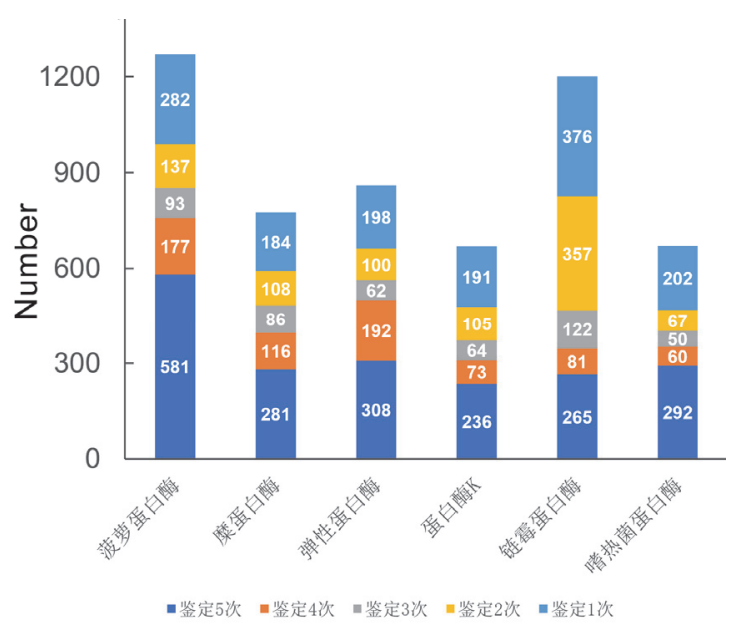

图 2 不同酶解时间所产生肽段被鉴定到的次数与数目

Figure 2 The frequencies and numbers of identified peptides produced at different digestion times

\section{3 基于非特异性蛋白酶的连续酶解}

选取 BSA 经六种蛋白酶一次酶解产生肽段数目最 多的结果分别与连续酶解的结果对比(图 3). 连续酶解 的方式所获得的肽段数目比一次酶解多 $16 \% \sim 131 \%$, 其中使用糜蛋白酶通过连续酶解的方式获得的肽段种 类是经过一次酶解所产生肽段种类的 2.3 倍. 由此可见, 通过连续酶解的方式产生的肽段种类数目多于一次酶 解，更多种类的肽段之间会有更多的重叠区域，有利于 蛋白质的序列拼接.

在序列覆盖率方面, 通过数据库搜索得到的肽段序 列覆盖率，仅有经菠萝蛋白酶酶解产生的肽段序列覆盖 率为 $100 \%$, 经过其余五种蛋白酶酶解产生的肽段覆盖 率为 $94.34 \%$ ～98.63\%, 虽然比特异性蛋白酶酶解所得 的序列覆盖率要高, 但仍达不到序列的全覆盖(表2). 而 使用连续酶解方式，经过这六种非特异性蛋白酶酶解后 肽段序列覆盖率都可达到 $100 \%$, 说明连续酶解的方式 有助于提高肽段的序列覆盖率. 


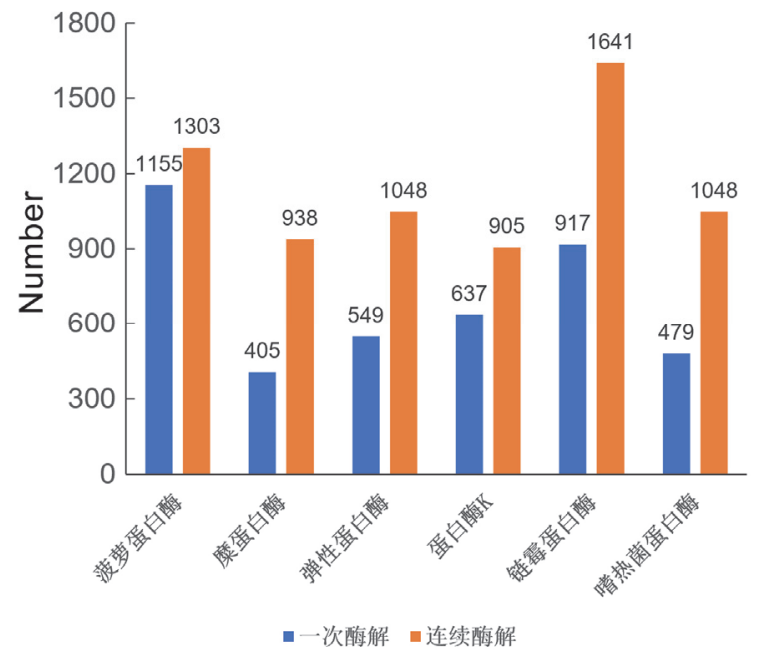

图 3 一次酶解和连续酶解方式所产生的肽段数目

Figure 3 Number of peptides produced by using one-time and continuous digestion

表 2 连续酶解与一次酶解的序列覆盖率 $(\%)$

Table 2 Sequence coverage of continuous digestion and one-time digestion $(\%)$

\begin{tabular}{ccccccc}
\hline 酶解方式 & $\begin{array}{c}\text { 菠萝蛋 } \\
\text { 白酶 }\end{array}$ & $\begin{array}{c}\text { 糜蛋白 } \\
\text { 酶 }\end{array}$ & $\begin{array}{c}\text { 弹性蛋 } \\
\text { 白酶 }\end{array}$ \\
\hline 一蛋白酶 $\mathrm{K}$ & $\begin{array}{c}\text { 链需蛋酶解 } \\
\text { 白酶 }\end{array}$ & $\begin{array}{c}\text { 嗜热菌 } \\
\text { 蛋白酶 }\end{array}$ \\
连续酶解 & 100 & 94.34 & 98.63 & 96.23 & 96.91 & 97.77 \\
\hline
\end{tabular}

\section{4 从头测序肽段的序列互补性分析}

使用 PEAKS (v8.5)软件对 BSA 经连续酶解产生的 肽段从头测序, 得到候选肽段序列. 再将每种蛋白酶酶 解后得到的候选序列与 BSA 序列进行匹配(图 4). 尽管 在数据库检索时 6 种蛋白酶酶解后的肽段的覆盖率都能 达到 $100 \%$, 但是由于肽段的从头测序的错误率高, 不 能对每个谱图进行准确测序, 因此 6 种蛋白酶酶解后的 从头测序得到的肽段序列都无法实现全覆盖, 不能仅通 过单一的蛋白酶酶解肽段拼接成完整的 BSA 全序列.

\section{5 蛋白质序列拼接}

使用我们开发的序列拼接算法, 将从头测序得到的
肽段序列进行拼接(图 5). 首先将从 PEAKS 输出的候选 序列(ALC $>50)$ 进行分解, 每个序列分解成若干个由 7 个氨基酸组成的子序列. 例如, 序列 LVVSTQTALA 是 由 10 个氨基酸组成的序列, 它被分解成 LVVSTQT (1 7)、VVSTQTA (2 8)、VSTQTAL (3 9) 和 STQTALA $(4 \sim 10)$ 四个子序列. 再统计所有候选序列分解成的每 一个子序列个数, 选出频数最高的子序列为种子序列. BSA 所产生的所有子序列中, 序列为 DSPDLPK 出现频 数最高为 2746 次, 故将其设定为种子序列, 再将其它所 有 7 个氨基酸组成的子序列与此种子序列做重叠分析, 在所有子序列中寻找包含种子序列 $\mathrm{N}$ 末端 6 个氨基酸的 序列(DSPDLP), C 端为 DSPDLP 的子序列有 DDSPDLP、 ADSPDLP 、 EDSPDLP 、 RDSPDLP 、 TDSPDLP 、 GDSPDLP、PDSPDLP、KDSPDLP 和 YDSPDLP, 以上 序列第一个氨基酸得分分别为 135558 、3536、2760、 2710、1424、1312、1297、1257 和 1188. 种子序列 DSPDLPK 向 $\mathrm{N}$ 端延伸的备选氨基酸残基为 D、A、E、 $R 、 T 、 G 、 P 、 K$ 和 $Y . D$ 的得分 135558 远高于次优氨 基酸 $\mathrm{A}$ 的得分 3536, 因此选取氨基酸残基 $\mathrm{D}$ 作为种子 序列 DSPDLPK 向 N 端延伸的氨基酸, 得到 8 个氨基酸 组成的序列 DDSPDLPK. 再在所有子序列中寻找包含 此 8 肽序列 $\mathrm{N}$ 端的 6 个氨基酸(DDSPDL)的序列, 找到 C 端为 DDSPDL 的子序列. 通过对子序列进行搜索, 发 现 8 肽序列可向 $\mathrm{N}$ 端延伸的候选氨基酸残基有 $\mathrm{K} 、 \mathrm{G} 、$

$H 、 V 、 A 、 L 、 M 、 Q$ 和 $P$, 其中 $K$ 的得分值是次优候选 氨基酸残基 $\mathrm{G}$ 的 15 倍, 故序列可延伸为 KDDSPDLPK. 本序列拼接算法可实现自动延伸, 依次在序列 $\mathrm{N}$ 端不断 增加氨基酸残基; 如果氨基酸残基的最优得分值没有达 到次优得分值的两倍及以上，则进行人工判断.

为了确定蛋白质 $\mathrm{N}$ 末端, 我们对蛋白质样品进行二 甲基化标记后再进行连续酶解, 酶解后产生的肽段的赖 氨酸和蛋白质 $\mathrm{N}$ 末端肽段的 $\mathrm{N}$ 端氨基酸会带有二甲基 化修饰. 在序列拼接组装过程中, 若遇到肽段 $\mathrm{N}$ 端的氨 基酸带有二甲基化修饰，则终止向 $\mathrm{N}$ 端延伸，即此序列 已延伸至蛋白质的 $\mathrm{N}$ 末端. 接着继续向此序列的 $\mathrm{C}$ 端延 伸，直到延伸的序列的分子量达到 BSA 分子量时，延伸

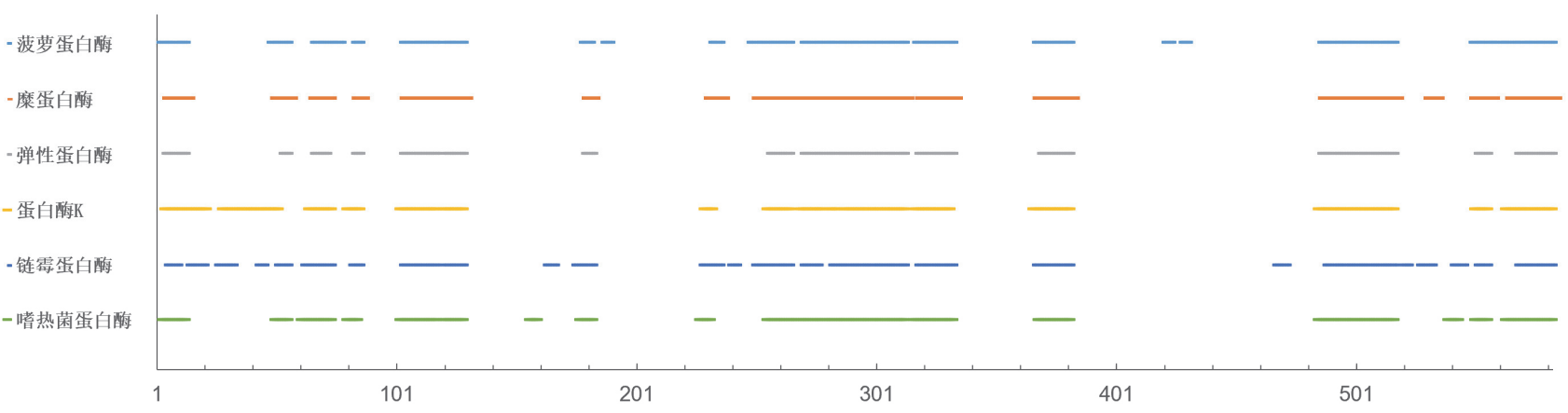

图 4 从头测序肽段的序列覆盖度

Figure 4 Sequence coverage of de novo sequencing peptides 


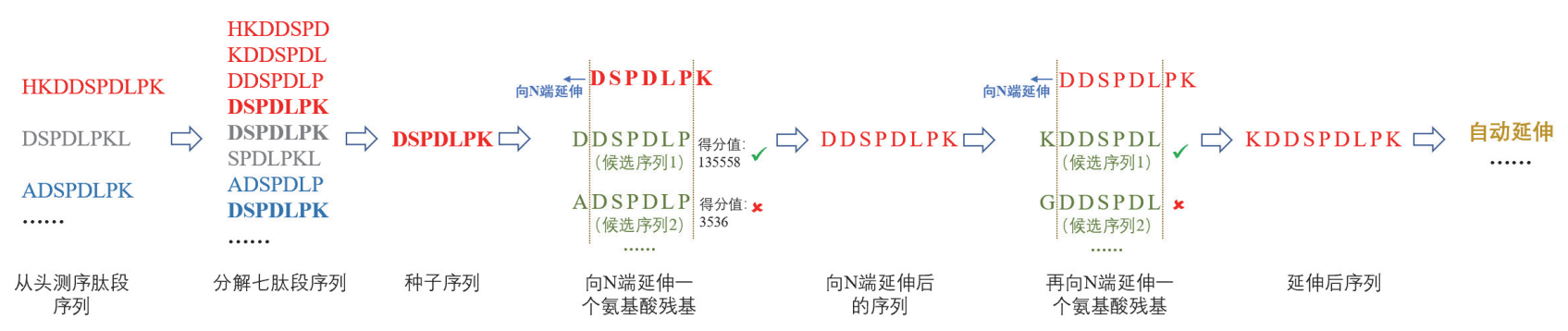

图 5 序列拼接流程

Figure 5 The diagram of sequence assembly

终止, 得到 BSA 的全部 583 个氨基酸的序列. 由于亮氨 酸与异亮氨酸分子量相同, 在 MS/MS 中无法区分, 所 以在测序过程中这两种氨基酸都被认为是亮氨酸.

\section{6 单克隆抗体的从头测序}

单克隆抗体赫赛汀是一种应用于乳腺癌的抗体药, 我们对赫赛汀的轻链和重链全序列进行测定. 单克隆抗 体经 6 种非特异性蛋白酶连续酶解, 使用高分辨率质谱 采集, 再通过 PEAKS 软件进行从头测序, 对测得的候 选肽段进行序列拼接, 得到重链和轻链的两个亚基的完 整序列, 测序覆盖率 $100 \%$, 对轻链测序准确度 $100 \%$, 重链测序准确度为 $99.7 \%$.

\section{3 结论}

本文使用非特异性蛋白酶通过连续酶解的方式对 蛋白质进行酶解, 获得种类更多的肽段, 相比于特异性 蛋白酶或非特异性蛋白酶的一次酶解, 显著提高序列覆 盖率以及序列的重叠度. 使用电动洜连续收集酶解产 物, 比起人工间隔定时收集酶解产物更简单、方便和高 效. 发展了蛋白质序列拼接算法, 可以将从头测序软件 获得的肽段序列拼接成完整蛋白质序列. 将此方法应用 于牛血清白蛋白和单克隆抗体赫赛汀的全序列测定, 在 不考虑亮氨酸和异亮氨酸的情况下, 对牛血清白蛋白和 赫赛汀轻链的测序准确度达到 $100 \%$, 赫赛汀重链的测 序准确度为 $99.7 \%$. 因此, 发展的基于非特异性蛋白酶 连续酶解的从头测序策略可应用于对未知序列蛋白质 的全序列测定或者对单克隆抗体序列进行表征.

\section{4 实验部分}

\section{1 仪器与试剂}

采用 EASY-nLCTM1200 色谱系统与 Orbitrap Q Exactive 质谱仪 (赛默飞, 美国) 构建分析系统 (Nano-RPLC-ESI-MS/MS), 用于对牛血清白蛋白(BSA) 和单克隆抗体赫赛汀酶解后的肽段进行富集与分析. 其 中 $\mathrm{C} 18$ 毛细管预柱和分离柱均为本实验室制作, Venusil XBP C18 硅胶填料(粒径 $5 \mu \mathrm{m}$, 孔径 $15 \mathrm{~nm}$ )购自博纳艾
杰尔(中国), Reprosil-PurC18-AQ 填料(粒径 $1.9 \mu \mathrm{m}$, 孔 径 $12 \mathrm{~nm}$ )购自 Dr. Maisch(德国). 真空浓缩仪购自赛默 飞科技公司(美国).

BSA、肤蛋白酶、二硫苏糖醇(DTT)、磑乙酰胺 (IAA)、盐酸胍、三羟甲基氨基甲烷(Tris)、糜蛋白酶、 弹性蛋白酶、嗜热菌蛋白酶、蛋白酶 $\mathrm{K}$ 和链霉蛋白酶、 $\alpha$-氰基-4-羟基肉桂酸 (CHCA)均购自西格玛-奥德里奇公 司(美国). 乙腈(ACN，色谱纯) 购自默克公司(德国). 菠萝蛋白酶购自百灵威公司(中国)。氯化钻 $\left(\mathrm{CaCl}_{2}\right.$, 分 析纯)购自科密欧公司(中国)。单克隆抗体赫赛汀由中国 计量科学研究院提供. 所有实验室用水均经过 Milli-Q(美国)系统处理.

\section{2 蛋白质酶解}

对 BSA 的一次酶解. 称取 $6 \mathrm{mg}$ BSA 粉末, 溶解在 $0.6 \mathrm{~mL} 6 \mathrm{~mol} / \mathrm{L}$ 盐酸胍溶液中, BSA 浓度为 $10 \mathrm{mg} / \mathrm{mL}$, 加入 $16 \mu \mathrm{L} 1 \mathrm{~mol} / \mathrm{L}$ DTT 在 $56{ }^{\circ} \mathrm{C}$ 水浴中加热 $1 \mathrm{~h}$, 再加 入 $40 \mu \mathrm{L} 1 \mathrm{~mol} / \mathrm{L} \mathrm{IAA}$ 室温避光 $30 \mathrm{~min}$ 烷基化, 将烷基 化后的 BSA 均分为 6 份, 分别使用以下缓冲液稀释 BSA 终浓度为 $1 \mathrm{mg} / \mathrm{mL}$, 并分别加入相应蛋白酶. $50 \mathrm{mmol} / \mathrm{L}$ Tris- $\mathrm{HCl}$ 溶液( $\mathrm{pH} 8.0$ ), 菠萝蛋白酶 $40 \mu \mathrm{g} ; 50 \mathrm{mmol} / \mathrm{L}$ Tris-HCl 溶液( $\mathrm{pH} 9.0$ ), 弹性蛋白酶 $40 \mu \mathrm{g} ; 100 \mathrm{mmol} / \mathrm{L}$ Tris- $\mathrm{HCl}$ 溶液(含 $10 \mathrm{mmol} / \mathrm{L} \mathrm{CaCl}_{2}, \mathrm{pH} 8.0$ ), 糜蛋白酶 40 $\mu \mathrm{g} ; 50 \mathrm{mmol} / \mathrm{L}$ Tris- $\mathrm{HCl}$ 溶液(含 $5 \mathrm{mmol} / \mathrm{L} \mathrm{CaCl}_{2}, \mathrm{pH} 7.5$ ), 蛋白酶 $\mathrm{K} 40 \mu \mathrm{g} ; 10 \mathrm{mmol} / \mathrm{L}$ Tris- $\mathrm{HCl}$ 溶液( $\mathrm{pH}$ 7.8), 链霉 蛋白酶 $40 \mu \mathrm{g} ; 50 \mathrm{mmol} / \mathrm{L}$ Tris- $\mathrm{HCl}$ 溶液(含 $0.5 \mathrm{mmol} / \mathrm{L}$ $\mathrm{CaCl}_{2}, \mathrm{pH}$ 8.0), 嗜热菌蛋白酶 $40 \mu \mathrm{g}$. 其中嗜热菌蛋白 酶的酶解反应在 $75{ }^{\circ} \mathrm{C}$ 水浴中进行, 其他酶解反应在 $37{ }^{\circ} \mathrm{C}$ 中进行. 为证明不同时间非特异性蛋白酶酶解产 生的肽段种类和数目不同, 对于 6 份非特异性蛋白酶酶 解反应，分别在 5、20、40、60、90、120、150、180、 $210 、 240 、 270 、 300 、 330 、 360$ 和 $420 \mathrm{~min}$ 时取出 $50 \mu \mathrm{L}$ 酶解后产物，立即使用 C18-Tip 除盐并冻干.

对 BSA 和单克隆抗体赫赛汀的连续酶解. 取 300 $\mu \mathrm{g}$ BSA 和 $300 \mu \mathrm{g}$ 赫赛汀, 分别加入 $0.6 \mathrm{~mL} 6 \mathrm{~mol} / \mathrm{L}$ 盐 酸胍溶液变性, 加入 $1 \mathrm{~mol} / \mathrm{L} \mathrm{DTT}$, 在 $56{ }^{\circ} \mathrm{C}$ 水浴加热条 件下还原 $1 \mathrm{~h}$, 冷却至室温后, 再加入 $1 \mathrm{~mol} / \mathrm{L} \mathrm{IAA}$ 避光 
$30 \mathrm{~min}$ 烷基化. 将以上烷基化后的 BSA 和赫赛汀分别 均分 6 份, 分别用以下缓冲液稀释 10 倍, 并加入非特异 性蛋白酶: $50 \mathrm{mmol} / \mathrm{L}$ Tris- $\mathrm{HCl}$ 溶液( $\mathrm{pH} 8.0)$, 菠萝蛋白 酶 $40 \mu \mathrm{g} ; 50 \mathrm{mmol} / \mathrm{L}$ Tris- $\mathrm{HCl}$ 溶液 $(\mathrm{pH} 9.0)$, 弹性蛋白酶 $40 \mu \mathrm{g} ; 100 \mathrm{mmol} / \mathrm{L}$ Tris- $\mathrm{HCl}$ 溶液(含 $10 \mathrm{mmol} / \mathrm{L} \mathrm{CaCl}_{2}$, $\mathrm{pH} 8.0$ ), 糜蛋白酶 $40 \mu \mathrm{g} ; 50 \mathrm{mmol} / \mathrm{L}$ Tris- $\mathrm{HCl}$ 溶液(含 5 $\mathrm{mmol} / \mathrm{L} \mathrm{CaCl} 2, \mathrm{pH} 7.5)$, 蛋白酶 $\mathrm{K} 40 \mu \mathrm{g} ; 10 \mathrm{mmol} / \mathrm{L}$ Tris- $\mathrm{HCl}$ 溶液 $(\mathrm{pH}$ 7.8), 链霉蛋白酶 $40 \mu \mathrm{g} ; 50 \mathrm{mmol} / \mathrm{L}$ Tris- $\mathrm{HCl}$ 溶液(含 $0.5 \mathrm{mmol} / \mathrm{L} \mathrm{CaCl}_{2}, \mathrm{pH} \mathrm{8.0)}$ ), 嗜热菌蛋白 酶 $40 \mu \mathrm{g}$. 将以上蛋白质与蛋白酶的混合物吸取至 500 $\mu \mathrm{L}$ 的进样针中, 并在进样针外部加上恒温加热套管, 其 中嗜热菌蛋白酶酶解温度设为 $75{ }^{\circ} \mathrm{C}$, 其余蛋白酶酶解 温度设定为 $37{ }^{\circ} \mathrm{C}$. 把进样针连同加热套管固定在电动 恒流洜上, 流速设为 $1.19 \mu \mathrm{L} / \mathrm{min}$, 反应时间为 $7 \mathrm{~h}$. 从进 样针流出的蛋白质与酶的混合溶液进入盛有饱和盐酸 胍溶液的离心管中. 使用 $\mathrm{C} 18$ 反相色谱柱除盐, 冻干. 对于抗体样品, 使用 $50 \mathrm{mmol} / \mathrm{L}$ 碳酸氢铵溶液复溶至 1 $\mu \mathrm{g} / \mu \mathrm{L}$, 加入底物与蛋白酶质量比为 $500: 1$ 的 PNGaseF 酶去 $\mathrm{N}$ 糖基化, $37{ }^{\circ} \mathrm{C}$ 水浴加热 $10 \mathrm{~h}$, 使用 $\mathrm{C} 18$ 反相色谱 柱除盐冻干保存. 使用 $0.1 \%$ 甲酸水溶液复溶至 $1 \mu \mathrm{g} / \mu \mathrm{L}$, $16000 \mathrm{~g}$ 离心后待质谱分析.

\subsection{MALDI-TOF-MS 分析}

取 $1 \mu \mathrm{L}$ 待分析物置于 MALDI 靶板上, 再点入 $1 \mu \mathrm{L}$ CHCA 基质 $(7 \mathrm{mg} / \mathrm{mL}$, 溶于含 $0.1 \%$ 三氟乙酸的 $60 \%$ 乙 腈溶液). 采用固体激光 Smart Beam 技术, 激光波长为 $355 \mathrm{~nm}$, 频率为 $200 \mathrm{~Hz}$, 加速电压为 $20 \mathrm{kV}$, 以及正离子 反射模式进行 MALDI-TOF MS 分析. 使用的分析软件 为 flexControl 和 flexAnalysis(布鲁克, 德国).

\subsection{RPLC-ESI-MS 分析}

采用 EASY-nLCTM1200 色谱系统和 Orbitrap Q Exactive 质谱仪分离和分析肽段, 每次载样 $1 \mu \mathrm{g}$, 每个 样品进行三次质谱重复实验. Nano-HPLC 的流动相 A 为 含有 $0.1 \%$ 三氟乙酸的 $2 \%$ 乙腈溶液; 流动相 $\mathrm{B}$ 为含有 $0.1 \%$ 三氟乙酸的 $98 \%$ 乙腈溶液. 流速为 $600 \mathrm{~nL} / \mathrm{min}$, 流 动相梯度: $0 \sim 1 \mathrm{~min}, 4 \% \mathrm{~B} ; 1 \sim 140 \mathrm{~min}, 4 \% \sim 30 \% \mathrm{~B}$; $140 \sim 165 \mathrm{~min}, 30 \% \sim 90 \% \mathrm{~B} ; 165 \sim 185 \mathrm{~min}, 90 \% \mathrm{~B}$.

Orbitrap Q Exactive 质谱参数设置如下: 使用数据 依赖型正离子模式采集数据, 喷雾电压为 $2.1 \mathrm{kV}$, 离子 传输管温度为 $260{ }^{\circ} \mathrm{C}$. 使用 Xcalibur 软件(2.3.0 版)将扫 描范围设置为 $m / z 350 \sim 1500$, 分辨率设置为 $7 \times 10^{4}$, 每 张一级谱的前 20 个母离子被选择去做二级谱, 离子最 大累计时间设为 $20 \mathrm{~ms}$, 自动增益控制设为 $3 \times 10^{6}$, 分 离窗口设为 $1.7 \mathrm{Da}$. 母离子采用高能碰撞碎裂 $(\mathrm{HCD})$ 模 式进行二级碎裂, 碎裂能量为 $30, \mathrm{MS} / \mathrm{MS}$ 分辨率设为 $7 \times 10^{4}$, 离子最大累计时间设为 $120 \mathrm{~ms}$, 动态排除时间 为 $12 \mathrm{~s}$, 自动增益控制设为 $1 \times 10^{6}$.

\section{5 数据库检索及从头测序}

原始 raw 文件由 Orbitrap Q Exactive 和 Velos 产生. 使用 $\mathrm{pFind}$ 软件(v 3.1)进行数据库检索, FASTA 数据库 为 UniProtKB ALBU_BOVIN. 检索参数设置为: 一级母 离子的质量容差设置为 $20 \mathrm{ppm}\left(20 \times 10^{-6}\right)$, 对于 Q Exactive 产生的 raw 文件二级碎片离子的质量容差设置为 $20 \mathrm{ppm}\left(20 \times 10^{-6}\right)$, 对于 Velos 产生的 raw 文件二级碎片 离子的质量容差设置为 $0.5 \mathrm{Da}$. 半胱氨酸的烷基化 $(+$ $57.02 \mathrm{Da})$ 设置为固定修饰，甲硫氨酸的氧化 $(+15.99$ $\mathrm{Da}$ 设置为可变修饰. 酶切特性选择 non-specific, 并允 许最多两个漏切位点. 其它设置均为软件默认.

使用 PEAKS (v 8.5)对 Orbitrap Q Exactive 产生的 raw 文件进行测序分析, 测序参数设置如下: 选择 Orbitrap 离子阱, 高能诱导碰撞碎裂模式(HCD), 蛋白酶 选择 none, 固定修饰与可变修饰与 pFind 数据库检索一 致. 母离子和碎片离子质量容差分别设为 $20.0 \mathrm{ppm}$ 和 $0.02 \mathrm{Da}$.

\section{6 拼接算法设计}

首先将经 PEAKS 软件测序得到的从头测序的肽段 序列分解，每张谱图鉴定的肽段序列分解成较短的七 肽, 如一个十肽可以被分解成四个七肽, 分别为十肽第 $1 \sim 7$ 氨基酸序列组成的肽段, 十肽第 $2 \sim 8$ 氨基酸序列 组成的肽段，十肽第 $3 \sim 9$ 氨基酸序列组成的肽段，十肽 第 4 10 氨基酸序列组成的肽段. 将所有从 PEAKS 测 序得到的肽段分解成七肽后，对于每一条唯一性的七 肽，统计其被鉴定到的次数，选择鉴定到次数最多的七 肽, 作为对蛋白质序列拼接的种子序列, 对其进行延伸.

将每个鉴定到的七肽序列与种子序列进行比较, 对 与种子序列末端个数(例如 6 个)氨基酸序列重叠的短肽, 统计短肽与种子序列重叠部分下一个氨基酸的种类及 其出现次数和得分值; 通过对所有短肽与种子序列重叠 部分下一个(种子序列待延伸位置)氨基酸的种类及其出 现次数和得分值进行综合打分，计算得到每种氨基酸的 总得分.

对种子序列待延伸位置每种氨基酸的总得分进行 比较(图 6); 如果某种氨基酸在短肽与种子序列末端数 个氨基酸重叠的情况下其得分值都大于次优氨基酸得 分的两倍以上. 则确定该氨基酸为种子序列待延伸位置 的最优氨基酸，并且按照该规则继续对种子序列进行延 伸, 直至无法继续延伸; 如果最优氨基酸的各项得分值 不大于次优氨基酸得分值的两倍, 则进行人工验证, 包 括察看相关谱图的质量以及观察氨基酸的选择对后续 拼接结果的影响(将排名靠前的氨基酸依次设为待延伸 位置的氨基酸，继续向后延伸，根据蛋白质序列的后续 拼接情况，选择合适的氨基酸). 在向 $\mathrm{N}$ 端延伸时，遇到 二甲基化标记的氨基酸则停止向 $\mathrm{N}$ 端延伸，继续向 $\mathrm{C}$ 端 延伸至直到序列的分子量达到待测蛋白分子量时，延伸 终止, 得到完整蛋白质序列. 


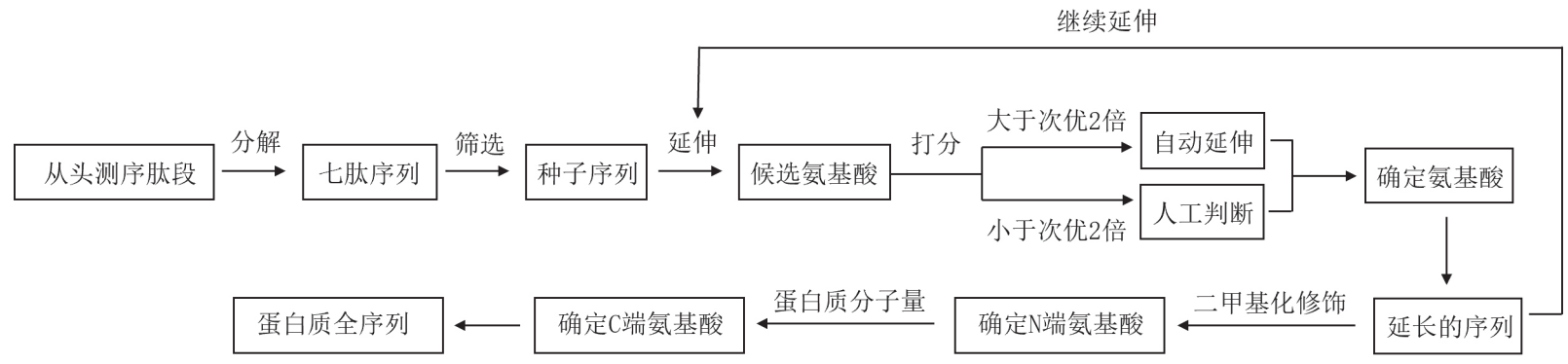

图 6 序列拼接算法

Figure 6 Sequence assembly algorithm

\section{致谢} 汀.

\section{感谢中国计量科学研究院提供的单克隆抗体赫赛}

\section{References}

[1] Galat, A. Arch. Biochem. Biophys. 1999, 371, 149.

[2] Jones, D. T.; Taylor, W. R.; Thornton, J. M. Bioinformatics 1992, 8, 275.

[3] Fowler, D. M.; Araya, C. L.; Fleishman, S. J.; Kellogg, E. H.; Stephany, J. J.; Baker, D.; Fields, S. Nat. Methods 2010, 7, 741.

[4] Beck, A.; Sanglier-CianféRani, S.; Van Dorsselaer, A. Anal. Chem. 2012, 84, 4637.

[5] Kelleher, N. L. Anal. Chem. 2004, 76, 197.

[6] Ge, Y.; Lawhorn, B. G.; Elnaggar, M.; Strauss, E.; Park, J.-H.; Begley, T. P.; Mclafferty, F. W. J. Am. Chem. Soc. 2002, 124, 672.

[7] Sun, R. X.; Luo, L.; Wu, L.; Wang, R. M.; Zeng, W. F.; Chi, H.; Liu, C.; He, S. M. Anal. Chem. 2016, 88, 3082.

[8] Seidler, J.; Zinn, N.; Boehm, M. E.; Lehmann, W. D. Proteomics 2010, 10, 634 .

[9] Zhang, Y.; Fonslow, B. R.; Shan, B.; Baek, M. C.; Yates, J. R. Chem. Rev. 2013, 113, 2343

[10] Liu, X.; Dekker, L. J. M.; Wu, S.; Vanduijn, M. M.; Pevzner, P. A.; Pa, P. J. Proteome Res. 2014, 13, 3241.

[11] Zhou, Y.; Xiao, Y. Acta Chim. Sinica 2018, 76, 177 (in Chinese). (周 怡青, 肖友利, 化学学报, 2018, 76, 177.)

[12] Tsiatsiani, L.; Heck, A. J. FEBS J. 2015, 282, 2612.

[13] Maccoss, M.; Mcdonald, W.; Saraf, A.; Sadygov, R.; Clark, J.; Tasto, J.; Gould, K.; Wolters, D.; Washburn, M.; Weiss, A.; Clark, J.; Yates, J. Proc. Natl. Acad. Sci. U. S. A. 2002, 99, 7900.

[14] Xu, T.; Wong, C. C. L.; Kashina, A.; Yates, J. R. Nat. Protoc. 2009, 4,325 .

[15] Allmer, J. Expert Rev. Proteomic. 2011, 8, 645.
[16] Mou, C.; Wang, H.; Zhou, P.; Hou, X. J. Comput. Appl. 2021, 1 (in Chinese). (牟长宁，王海鹏，周丕宇，侯金行，计算机应用，2021, 1.)

[17] Yang, C.; Liu, J.; Zhang, W.; Shan, Y.; Dai, Z.; Zhang, L.; Zhang, Y. Chin. J. Anal. Chem. 2021, 49, 366 (in Chinese). (杨超, 刘健慧，张 玮杰, 单亦初, 戴忠鹏, 张丽华, 张玉奎, 分析化学, 2021, 49, 366.)

[18] Ma, B.; Zhang, K.; Hendrie, C.; Liang, C.; Li, M.; Doherty - Kirby, A.; Lajoie, G. Rapid Commun. Mass Spectrom. 2003, 17, 2337.

[19] Zhang, J.; Xin, L.; Shan, B.; Chen, W.; Xie, M.; Yuen, D.; Zhang, W.; Zhang, Z.; Lajoie, G. A.; Ma, B. Mol. Cell. Proteomics 2012, 11, M111.010587.

[20] Chi, H.; Sun, R.-X.; Yang, B.; Song, C.-Q.; Wang, L.-H.; Liu, C.; Fu, Y.; Yuan, Z.-F.; Wang, H.-P.; He, S.-M. J. Proteome Res. 2010, 9, 2713.

[21] Yang, H.; Chi, H.; Zeng, W.-F.; Zhou, W.-J.; He, S.-M. Bioinformatics 2019, 35, i183.

[22] Yang, H.; Chi, H.; Zhou, W.-J.; Zeng, W.-F.; He, K.; Liu, C.; Sun, R.-X.; He, S.-M. J. Proteome Res. 2017, 16, 645.

[23] Yang, H.; Li, Y.-C.; Zhao, M.-Z.; Wu, F.-L.; Wang, X.; Xiao, W.-D.; Wang, Y.-H.; Zhang, J.-L.; Wang, F.-Q.; Xu, F. Mol. Cell. Proteomics 2019, 18, 773 .

[24] Frank, A.; Pevzner, P. Anal. Chem. 2005, 77, 964.

[25] Frank, A. M.; Savitski, M. M.; Nielsen, M. L.; Zubarev, R. A.; Pevzner, P. A. J. Proteome Res. 2007, 6, 114.

[26] Ma, B. J. Am. Soc. Mass Spectrom. 2015, 26, 1885.

[27] Cheng, X.; Yang, X.; Li, J. Genomics and Applied Biology 2020, 39, 3431 (in Chinese). (成茜, 杨篠韵, 李婧, 基因组学与应用生物学, 2020, 39, 3431.)

[28] Muth, T.; Renard, B. Y. Brief. Bioinform. 2018, 19, 954.

[29] Lu, B.; Chen, T. J. Comput. Biol. 2003, 10, 1-12s.

[30] Savidor, A.; Barzilay, R.; Elinger, D.; Yarden, Y.; Levin, Y. Mol. Cell. Proteomics 2017, 16, 1151.

[31] Tran, N. H.; Rahman, M. Z.; He, L.; Xin, L.; Shan, B.; Li, M. Sci. Rep. 2016, 6, 1.

[32] Yang, C.; Liu, J.; Hu, Y.; Dai, Z.; Zhang, Y. J. Sep. Sci. 2020, 43, 3665 .

(Cheng, B.) 